\title{
Effective Strategies for ROI and Image Matching
}

\author{
Dr. Khaled M. G. Noaman \\ Associate Professor \\ Department of Distance Learning \\ Deanship of E-Learning and Distance Learning, \\ Jazan University \\ Jazan, Kingdom of Saudi Arabia (KSA)
}

\author{
Dr. Jamil Abdulhamid M. Saif \\ Associate Professor \\ Information Systems Department \\ Community College of Bisha, Bisha University \\ Bisha, Kingdom of Saudi Arabia (KSA)
}

\begin{abstract}
The paper presents an exceptional four matching strategies: systematic, random, gradient and simulated annealing using diferent metrics. We consider two kinds of image matching algorithms. The first one oriented on the whole image matching where we compare corresponding pixels or chosen image characteristics. The second one is oriented on finding the region in the target image (region of interest ROI), which match best the ROI given in the template image. For our experiments we take the list of target images, directly from the atlas, and a subset of these images as the template images.
\end{abstract}

Keywords—systematic; random; gradient; simulated annealing

\section{INTRODUCTION}

Presently digital image processing has a broad spectrum of applications, such as multimedia systems, business systems, monitoring and inspection systems, archiving systems. Architectures of such systems are much complex (see Fig. 1). In spite of digitisation, storage, transmission, and display operations, extra functions are considered. They are as follows: image data compression and representation, image enhancement and reconstruction, image indexing retrieval and matching, etc. and they are executed on application oriented servers.

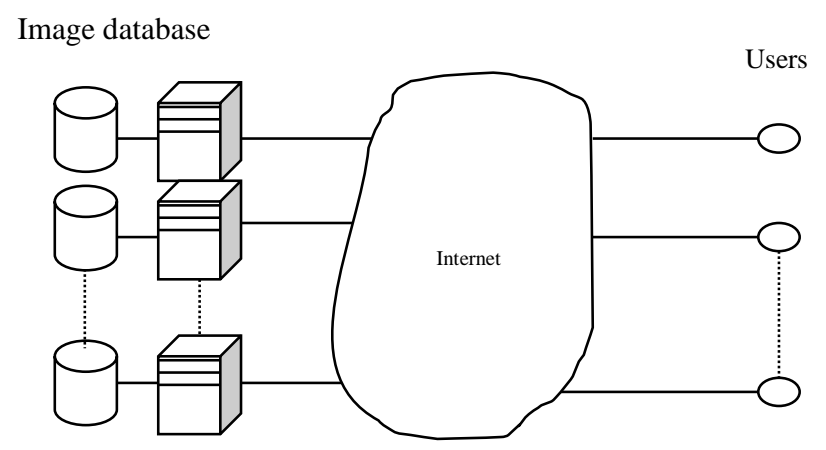

Fig. 1. Modem digital image processing system

In medical applications clinical diagnosis as well as evaluation of therapy is often supported by image processing systems. Such techniques as SPECT (Single Photon Emission Computed Tomography), PET (Positron Emission Tomography), MRS (Magnetic Resonance Spectroscopy), MRI (Magnetic Resonance Imaging), or ultrasound and X-ray scanning are largely used and developed $[1,2]$. The Internet creates a new possibilities for medicine diagnosis. Application of multiedia systems provides a real-time medical monitoring multi-party consultations and distance collaborations. Examples of such solutions are the following systems:

1) MedNet - used in brain surgery [3],

2) Telematic microscopy system used in diagnostics of histopathology [4],

3) Medinet - used in diagnostics of teleradiology [5].

The rest of this paper is organized as follows: section 2 presents the matching problem and defines the similarity for Whole image and region-based image matching. In section 3, experimental results are presented and discussed, also the effectiveness of our proposed method are discussed. The conclusion and the proposal of future works are given in Section4.

\section{IMAGe MAtching PRoblem}

A digital image $(\mathrm{I}(\mathrm{m}, \mathrm{n}), \mathrm{m}, \mathrm{n}$-integers $)[6,7,8]$ is usually the result of discretization process of a continuous image function $\mathrm{I}(\mathrm{x}, \mathrm{y}) \mathrm{x}, \mathrm{y} \in \mathrm{R}$, and it is stored in a computer memory as a two dimensional array $\mathrm{A}$, where $\mathrm{A}=[\mathrm{A}(\mathrm{m}, \mathrm{n})]$, $\mathrm{m}=1,2, \ldots, \mathrm{M}, \mathrm{n}=1,2, \ldots ., \mathrm{N}$; i.e:

We limit our considerations to the discrete image describing by two dimensional array A. However, other image dimensions can be taken into account (1D, 3D, ..., etc.) $[9,10]$, depending on what kind of imaging systems is used to create digital images. Each $\mathrm{A}(\mathrm{m}, \mathrm{n})$ element of the array A corresponds to a pixelwhich describes some properties of the image. We can use many shades of grey typically 16 or 256 to represent the pixels. However, grey scanning requires larger amounts of memory. In spite of a greyscale images are simple and have less information in comparison to colour images. It is possible to construct all visible colours by combining the three primary colours: red, green and blue (RGB colour image).

\section{A. Image Matching Algorithms and related definitions}

Theimage matching algorithms for the compared images or ROIs regarding the accuracy can be evaluated by the similarity degree, therefore we give the following definition that is needed for the matching problem.

\section{Definition 2.1.}

Let be given matrix A1 representing a template image I1 and matrix A2 representing a target image I2. For images I1 and $\mathrm{I} 2$ the following three cases should be considered:

1) Images are the same $(A 1=A 2)$ if and only if similarity criteria $\operatorname{SC}(A 1, A 2)=1$. 
2) Images II and I2 are similar if and only if $\Delta \leq S C$ (A1, A2) $<1$.

3) Images $I 1$ and $I 2$ are different if and only if $0 \leq S C(A 1$, A2) $<\Delta$.

Similarity criteria SC and threshold $\Delta(\mathrm{SC} \varepsilon<0,1>$ and $\Delta$ $\varepsilon<0,1>$ ) can be chosen arbitrarily for each class of matching algorithms.

In case of pixel to pixel comparison [11,12], we can define similarity criterion $\mathrm{SC}(\mathrm{Ik}, \mathrm{Ik}+1)$ as the following formula:

$$
M S=S C\left(I^{k}, I^{k+1}\right)={\sqrt{\sum_{i=1}^{M} \sum_{j=1}^{N}\left[A^{k}(i, j)-A^{k+l}(i, j)\right.}}^{2}
$$

where $A^{\mathrm{x}}(\mathrm{i}, \mathrm{j})$ is the pixel digital value for $\mathrm{x}^{\text {th }}$ image, it can be referred either to the whole image or to its ROI (see Fig. 2, 3 ). In many cases the similarity degree MS is higher for ROI than for the whole images. In case of ROI the similarity criterion should be suitable modified (i.e. proper pixels are only compared).

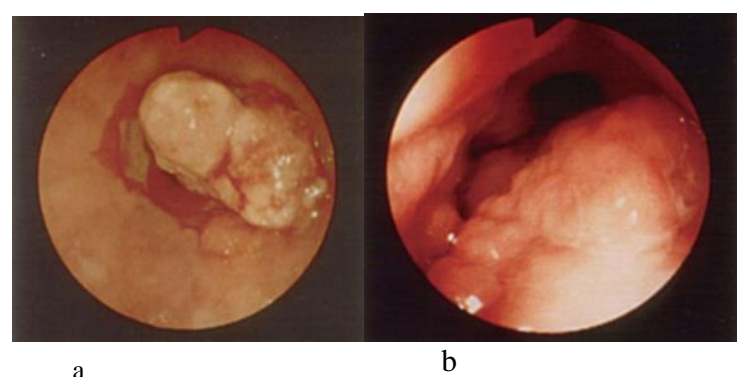

similarity degree $=0.8394$

Fig. 2. Example of matching two images, a) template, b) target

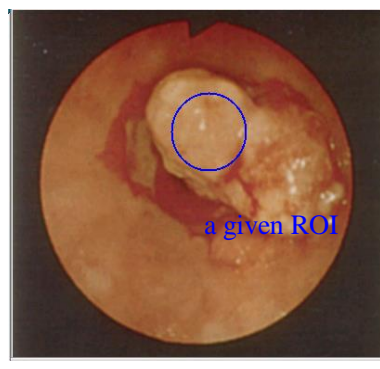

$\mathrm{a}$

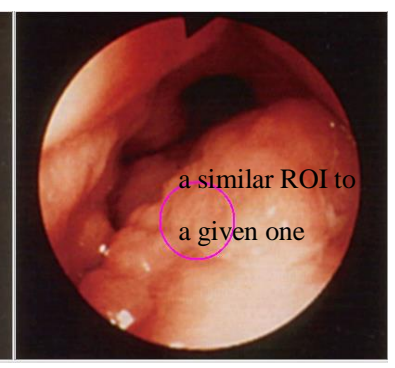

b

Fig. 3. Example of matching two ROI, a) template, b) target

\section{B. Image Matching Algorithms}

To solve the matching problem we propose four algorithm[13], systematic (lexicographical) searching, gradient searching, random searching and simulated annealing searching, that define the methods of searching the ROI in the target image that is best match the one specified in the template image. In systematic searching algorithm an initial location of the ROI is assumed to be on the left top corner of the target image, the the center of the ROI is moved from left to right and up to down in the target image with specific step of pixels, for each location of the ROI the relative similarity degree is calculated, at the end the location with the best similarity degree is pointed out as the best solution. Gradient searching algorithm can assume a random choice of the initial location of the ROI in the target image, next we calculate the step and the direction of the ROI movement, to find the best matching location we decrease the step twice in each iteration that returned the optimal location, and from that location we repeat this process of searching we get the best matching location. In random searching we determine only the number of iterations and every iteration the location of the ROI is randomly selected, after such process the optimal location with the best matching similarity of the ROI in the target image is returned. Finally in simulated annealing searching algorithm also the initial location of the ROI in the target image is selected randomly with a given number of iterations and with high starting temperature which is reduced in each iteration according to the annealing scheme, the location of the ROI in each location then is changed with probability determined by the generation function and the similarity degree is calculated for the new location with probability determined by the acceptance. After reaching the maximum iteration, we choose the optimal solution the found solutions.

\section{EXPERIMENTAL RESULTS AND DISCUSSION}

Experiments are carried out for endoscopic and the obtained results discussed in order to evaluate the different searching procedures, that helps choosing the best procedure of matching the whole endoscopic image, as well as ROI searching. The representative benchmarks for illustration of our considerations is presented in table 1 ., where there are different endoscopic images (size of $800 \times 720$ pixels), chosen from endoscopic atlas [14] among 1500 available images. We concentrate on stomach diseases, and include five images (from 1 to 5), corresponding to healthy patients, next nine images (from 6 to 14) contains some changes referring to typical (representative) stomach diseases. The last five images from 15-19 represent similar changes regarding to appearance.

TABLE I. DETAILED DESCRIPTION OF THE ENDOSCopic IMAGES BELONGING TO THE TEST BENCHMARK

\begin{tabular}{|c|c|c|}
\hline $\begin{array}{c}\text { The number of } \\
\text { image in Fig. 5.1 }\end{array}$ & $\begin{array}{c}\text { Its atlas } \\
\text { number }\end{array}$ & $\begin{array}{l}\text { Description of changes in the } \\
\text { endoscopic images }\end{array}$ \\
\hline 1 & $1 \_24$ & Formix fundus, healthy \\
\hline 2 & $1 \_25$ & Corpus venriculi, healthy \\
\hline 3 & $1 \_26$ & Corpus ventriculi, healthy \\
\hline 4 & $1 \_27$ & Antrum, anqulus, healthy \\
\hline 5 & $1 \_32$ & Antrum, healthy \\
\hline 6 & $6 \_15 \_a$ & Ventriculus, cancer \\
\hline 7 & $6 \_18 \_a$ & Ventriculus, cancer \\
\hline 8 & $6 \_18 \_b$ & Ventriculus, cancer \\
\hline 9 & $6 \_18 \_c$ & Ventriculus, cancer \\
\hline 10 & $6 \_23$ & Ventriculus, cancer \\
\hline 11 & $6 \_25 \_b$ & Ventriculus, cancer \\
\hline 12 & $6 \_5$ & Corpus, cancer \\
\hline 13 & $6 \_8 \_a$ & Corpus, cancer \\
\hline 14 & $6 \_8 \_b$ & Corpus, cancer \\
\hline 15 & $6 \_27$ & Fundus ventriculus, cancer \\
\hline 16 & $6 \_30 \_b$ & Fundus ventriculus, cancer \\
\hline 17 & $6 \_32 \_c$ & Corpus ventriculus, cancer \\
\hline 18 & $6 \_3 \_a$ & Corpus, antrum, cancer \\
\hline 19 & $6 \_6$ & Cardia, cancer \\
\hline
\end{tabular}




\section{A. Image and ROI matching strategies}

We consider two kinds of image matching algorithms. The first one oriented on the whole image matching where we compare corresponding pixels or chosen image characteristics.

The second one is oriented on finding the region in the target image which matches best the ROI given in the template image. In our experiments we take the list of target images, directly from the atlas, and a subset of these images as the template images. In case of simple matching criteria (MS formula (2.1), IF - formula [13] the similarity degree for the whole image gives value 1 for the same image. Table 2. confirms such cases.

Let consider ROI - oriented matching for the same set of template and target images. Note that even in case of the same compared images values of similarity degree are not equal to 1. Moreover, we can find higher value of these criteria for different images than for the same images see the first and the column of Table 3 . However, it does not mean that this kind of matching is not practically acceptable.

TABLE II. THE Similarity Degree VALUeS For the Whole IMAGE MATCHING

\begin{tabular}{|c|c|c|c|c|c|c|}
\hline $\begin{array}{r}\text { No of template } \\
\text { images }\end{array}$ & $\mathbf{1}$ & $\mathbf{2}$ & $\mathbf{7}$ & $\mathbf{1 1}$ & $\mathbf{1 6}$ & $\mathbf{1 7}$ \\
$\begin{array}{c}\text { No of target } \\
\text { images }\end{array}$ & & & & & & \\
\hline 1 & $\mathbf{1 . 0 0 0 0}$ & 0.7910 & 0.6744 & 0.7353 & 0.8140 & 0.6342 \\
\hline 2 & 0.7910 & $\mathbf{1 . 0 0 0 0}$ & 0.7529 & 0.7583 & 0.7792 & 0.7437 \\
\hline 3 & 0.7715 & 0.8285 & 0.7465 & 0.7512 & 0.7945 & 0.7461 \\
\hline 4 & 0.7479 & 0.8515 & 0.7985 & 0.7351 & 0.7617 & 0.7591 \\
\hline 5 & 0.8185 & 0.8385 & 0.7415 & 0.7460 & 0.8062 & 0.7311 \\
\hline 6 & 0.6928 & 0.7135 & 0.7637 & 0.7617 & 0.7734 & 0.7442 \\
\hline 7 & 0.6744 & 0.7529 & $\mathbf{1 . 0 0 0 0}$ & 0.7421 & 0.7211 & 0.7845 \\
\hline 8 & 0.7142 & 0.7805 & 0.7859 & 0.7695 & 0.7562 & 0.7649 \\
\hline 9 & 0.6677 & 0.7925 & 0.7453 & 0.6623 & 0.6703 & 0.7463 \\
\hline 10 & 0.7232 & 0.7870 & 0.6684 & 0.7332 & 0.7623 & 0.7463 \\
\hline 11 & 0.7353 & 0.7583 & 0.7421 & $\mathbf{1 . 0 0 0 0}$ & 0.7949 & 0.7133 \\
\hline 12 & 0.7429 & 0.8167 & 0.7500 & 0.7342 & 0.7790 & 0.7706 \\
\hline 13 & 0.6685 & 0.7579 & 0.7860 & 0.7338 & 0.7417 & 0.8343 \\
\hline 14 & 0.7964 & 0.8451 & 0.7704 & 0.7711 & 0.8210 & 0.7728 \\
\hline 15 & 0.8016 & 0.8232 & 0.7691 & 0.7739 & 0.8049 & 0.7482 \\
\hline 16 & 0.8140 & 0.7792 & 0.7211 & 0.7949 & $\mathbf{1 . 0 0 0 0}$ & 0.7044 \\
\hline 17 & 0.6342 & 0.7437 & 0.7845 & 0.7133 & 0.7044 & $\mathbf{1 . 0 0 0 0}$ \\
\hline 18 & 0.6478 & 0.7337 & 0.7873 & 0.6771 & 0.6847 & 0.7252 \\
\hline 19 & 0.7520 & 0.7841 & 0.7450 & 0.7753 & 0.8010 & 0.7500 \\
\hline
\end{tabular}

TABLE III. SIMILARITY DEGREE VALUES FOR ROI - ORIENTED MATCHING

\begin{tabular}{|c|c|c|c|c|c|c|}
\hline $\begin{array}{r}\begin{array}{r}\text { No of } \\
\text { template } \\
\text { images }\end{array} \\
\text { No of target } \\
\text { images }\end{array}$ & 1 & 2 & 7 & 11 & 16 & 17 \\
\hline 1 & 0.9082 & 0.8465 & 0.7406 & 0.7566 & 0.7600 & 0.7739 \\
\hline 2 & 0.9068 & 0.9689 & 0.8229 & 0.8755 & 0.8177 & 0.8804 \\
\hline 3 & 0.8553 & 0.7815 & 0.7982 & 0.8021 & 0.7820 & 0.8159 \\
\hline 4 & 0.8598 & 0.8828 & 0.8513 & 0.8612 & 0.8516 & 0.8752 \\
\hline 5 & 0.8468 & 0.9031 & 0.7488 & 0.7721 & 0.7768 & 0.7703 \\
\hline 6 & 0.8710 & 0.8400 & 0.8681 & 0.8895 & 0.8903 & 0.9046 \\
\hline 7 & 0.8696 & 0.8289 & 0.9514 & 0.9101 & 0.8708 & 0.9153 \\
\hline 8 & 0.8799 & 0.8163 & 0.8751 & 0.9002 & 0.8893 & 0.9282 \\
\hline 9 & 0.8860 & 0.8252 & 0.9297 & 0.8807 & 0.8812 & 0.9041 \\
\hline 10 & 0.8837 & 0.8299 & 0.8703 & 0.8839 & 0.8715 & 0.9004 \\
\hline 11 & 0.9089 & 0.8086 & 0.8616 & 0.9262 & 0.8830 & 0.9401 \\
\hline 12 & 0.8619 & 0.8337 & 0.8878 & 0.9097 & 0.8907 & 0.9359 \\
\hline 13 & 0.8549 & 0.8253 & 0.8804 & 0.8978 & 0.8852 & 0.9104 \\
\hline 14 & 0.8671 & 0.8455 & 0.8168 & 0.8849 & 0.8737 & 0.8566 \\
\hline 15 & 0.8500 & 0.8227 & 0.7825 & 0.8117 & 0.8021 & 0.8238 \\
\hline 16 & 0.8661 & 0.8164 & 0.8480 & 0.8614 & 0.9374 & 0.8698 \\
\hline 17 & 0.8463 & 0.8213 & 0.9023 & 0.9033 & 0.8900 & 0.9375 \\
\hline 18 & 0.8657 & 0.8073 & 0.9195 & 0.9183 & 0.8819 & 0.9263 \\
\hline 19 & 0.8954 & 0.8276 & 0.8842 & 0.8882 & 0.8559 & 0.8989 \\
\hline
\end{tabular}

\section{B. Evaluation of searching procedures}

We consider four sequential procedures: systematic, random, gradient and simulation annealing defined in [13]. They operate only on pairs of target/template images where ROI's are determined by experts. In our experiments we assume that the target image is the same as the template one, but without ROI. We made many such experiments, but representative results are shown in Table 4. and Fig. 4., the best results we obtained for simulation annealing procedure, then for gradient procedure, we also note that random and systematic procedures give nearly the same level of the mean accuracy, however they are a bit a lower than the first two procedures.

TABLE IV. THE MEAN IMAGE MATCHING ACCURACY OF SEARCHING PROCEDURES

\begin{tabular}{|c|c|c|c|c|}
\hline $\begin{array}{c}\text { Searching } \\
\text { procedure }\end{array}$ & Systematic & Gradient & Random & $\begin{array}{c}\text { Simulated } \\
\text { annealing } \\
\text { No of compared } \\
\text { image }\end{array}$ \\
\hline 4 & 0.7446 & 0.6729 & 0.8416 & 0.8721 \\
\hline 5 & 0.6297 & 0.5815 & 0.5851 & 0.7792 \\
\hline 10 & 0.7688 & 0.7648 & 0.7556 & 0.8426 \\
\hline 16 & 0.8407 & 0.8153 & 0.8276 & 0.9210 \\
\hline 17 & 0.8265 & 0.9508 & 0.8459 & 0.8556 \\
\hline 19 & 0.7827 & 0.8910 & 0.7448 & 0.7920 \\
\hline Mean value & 0.7655 & 0.7794 & 0.7648 & 0.8437 \\
\hline
\end{tabular}




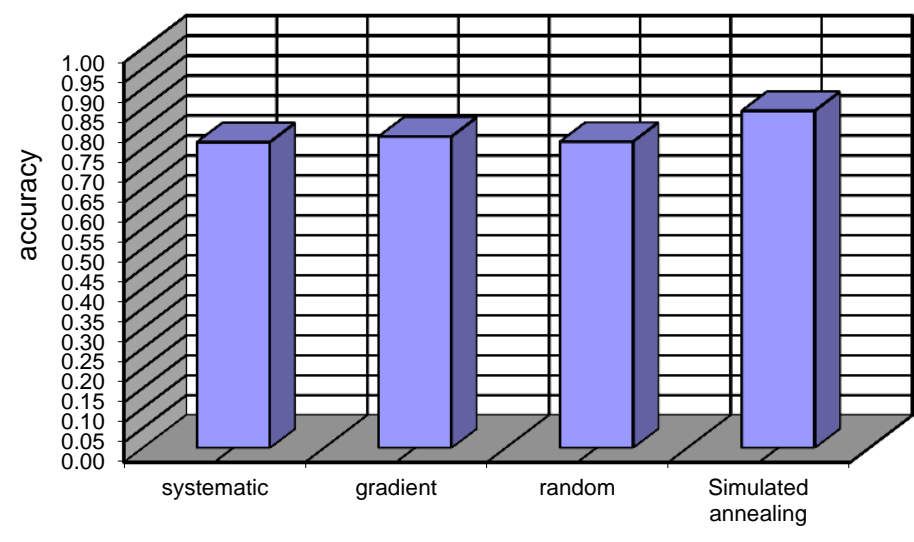

searching procedures

Fig. 5. The mean image matching accuracy of searching procedures

\section{CONCLUSION}

In our paper four searching procedures were investigated and analyzed for the endoscopic images. Such images are very heavy for analysis owing to some deformations made during their registrations. Therefore we decide to construct four such algorithms different from each other by use of systematic random, gradient and simulation annealing searching methods. All versions are so flexible that can be tune to improve quality of searching and matching in accordance to specific features of endoscopic images.

For future work further improvement of the algorithms should be done. As well as parallelization of searching procedures will be presented and the version parallel matching algorithms will be described, analyzed and evaluated.

\section{REFERENCES}

[1] Elsen P. A. V. D., Pol Evert-Jan D., Viergever M. A.: Medical Image Matching - A Review with Classification. IEEE Trans. on Engineering in Medicine and Biology, March 1993.

[2] ZulaikhaBeevi S. And Sathik M. M. :An Effective Approach for Segmentation of MRI Images: Combining Spatial Information with Fuzzy C-Means Clustering, European Journal of Scientific Research,EuroJournals Publishing, Inc. 2010,http://www.eurojournals.com/ejsr.htm.
[3] Simon R., Krieger D., Znati T., Lofink R., Sclabassi R.J.: Multimedia MedNet - A Medical Collaboration and Consultation System. IEEE Computer, May 1995.

[4] Sacile R., Ruggiero C., Lombardo G., Nicolo G., Wolf B., Petersen R.I.: Collaborative Diagnosis over the Internet: A Working Experience. IEEE Internet Computing, November 1999.

[5] Abrado A., Cassini L.: Embedded Java in a Web-Based Teleradiology System. IEEE Internet Computing, May 1998.

[6] Chanda B. And Majumder D. D.: Digital Image Processing and Analysis, Prentice Hall, 2003.

[7] Gonzalez C. and Woods R. E. :. Digital Image Processing, Second Edition, Prentice Hall, 2002.

[8] Umbaugh S. E. Computer Vision and Image Processing: A Practical Approach Using CVIP tools, Prentice Hall, 1998.

[9] Anil K. J.: Fundamentals of Digital Image Processing. Prentice-Hall International, 1989.

[10] Johnson A., Hebert M.:Using spin images for efficientobject recognition in cluttered 3D scenes,. IEEE Trans. On PAMI , 21(5):433-449, 1999.

[11] Grauman K. and Darrell T. :Efficient Image Matching with Distributions of Local Invariant Features, Proceedings of the IEEE conference on computer Vision and Pattern Recognition (CVPR), June 2005.

[12] Manimala S. and Hemachandran K, "Image Retrieval-Based on Color Histogram and Performance Evaluation of similarity Measurement", Assam University Journal of science \& Technology, Vol. 8 Number II 94-104, 2011.

[13] Saif J.: Sequential \& parallel image matching algorithms for endoscopic recommendations, PHD Thesis WETI2003, Gdansk, April 2004.

[14] Silverstein F.E., Tytgat G.N.J.: Gastrointestinal Endoscopy. Times Mirror Int. Publisher Limited, 1997.

\section{AUTHOR PROFILES}

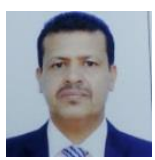

Khaled M. G. Noaman received the $\mathrm{PhD}$ degree in Artificial Intelligent (artificial neural networks) from Wroclaw University of Technology, Wroclaw, Poland, in 1999. In July 2006 he got promoted to associate professor at the Faculty of Computer Science and Information Technology, Sana'a University, Sana'a, Yemen. From 2012 till now he is working in the department of Distance Learning, Deanship of E-Learning and Distance Learning, Jazan University, Jazan, Kingdom of Saudi Arabia.

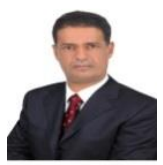

Jamil A. M. Saif received the Msc degree in telecommunication engineering, from the Department of telecommunication, Faculty of Automatic Control, Electronics and Computer Science, Silesian University of Technology, Gliwice, Poland, in 1993, the PhD degree in Computer Science from the Department of Computer Architecture, Faculty of Electronics, telecommunications and Informatics, Gdansk University of Technology, Gdansk, Poland, in 2004. In May 2012 he got promoted to associate professor at the Faculty of Computer Science and Engineering, Hodeidah University, Hodeidah, Yemen. From 2013 till now he is working in the Department of Information Systems, Community College of Bisha, King Khalid University, Kingdom of Saudi Arabia. 